\title{
Article \\ Homelessness and Research: Methodological Obstacles and Lessons Learned from a Psychological Study in Parisian Homeless Services
}

\author{
Gaëtan Chevreau ${ }^{1, *}$, Claire Vallat-Azouvi ${ }^{1}$, Marta Coll ${ }^{2}$, Frédéric Barbot ${ }^{3}$ and Marie-Carmen Castillo ${ }^{1}$ \\ 1 Laboratoire de Psychopathologie et Neuropsychologie, Université Paris 8 Vincennes-Saint-Denis, \\ 93200 Saint-Denis, France; claire.vallat-azouvi@univ-paris8.fr (C.V.-A.); \\ marie-carmen.castillo02@univ-paris8.fr (M.-C.C.) \\ 2 Lits Halte Soins Santé (LHSS) Maubeuge, Groupe Sos Solidarité, 75011 Paris, France; \\ marta.coll@groupe-sos.org \\ 3 Centre d'Investigation Clinique (Inserm CIC 1429), Université Paris-Saclay, \\ AP-HP Hôpital Raymond Poincaré, 92380 Garches, France; frederic.barbot@aphp.fr \\ * Correspondence: gaetanchevreau@gmail.com
}

\section{check for}

updates

Citation: Chevreau, G.;

Vallat-Azouvi, C.; Coll, M.; Barbot, F.;

Castillo, M.-C. Homelessness and

Research: Methodological Obstacles

and Lessons Learned from a

Psychological Study in Parisian

Homeless Services. Psych 2021, 3 ,

184-196. https://doi.org/10.3390/

psych3020016

Academic Editors: Mosad Zineldin and Graham Pluck

Received: 28 February 2021

Accepted: 3 June 2021

Published: 7 June 2021

Publisher's Note: MDPI stays neutra with regard to jurisdictional claims in published maps and institutional affiliations.

Copyright: (c) 2021 by the authors. Licensee MDPI, Basel, Switzerland. This article is an open access article distributed under the terms and conditions of the Creative Commons Attribution (CC BY) license (https:// creativecommons.org/licenses/by/ $4.0 /)$.

\begin{abstract}
Homelessness, defined as a lack of appropriate, stable, and permanent housing, is a common issue in many societies and is linked to both structural and individual factors. These factors include psychological mechanisms and disorders which can trigger or worsen already precarious situations. In order for these factors to be taken into account in social rehabilitation programs, they need to be precisely described. However, at present, studies in this field are lacking in France. Despite homelessness being an issue across the country, few studies have evaluated the underlying psychological or neuropsychological mechanisms. More data are needed, not only to provide an accurate description of the situation in France, but also to ensure that foreign observations and interventions are relevant for application to the homeless population. In order to achieve this, more quantitative and qualitative data and investigative methodologies and studies are needed. Sharing experience and methods within the scientific community is one way to support further research, particularly in complex domains such as homelessness. At the moment, only a few such papers have been published. In this paper, we share our experiences from a research project that started in 2020 (currently unpublished) on the prevalence of cognitive disorders among homeless service users in Paris. We describe the exploratory phase of our project, obstacles encountered during the implementation of the study, including how we dealt with ethical issues, and data collection. We end the paper with recommendations for future psychological studies on homelessness.
\end{abstract}

Keywords: homelessness; ethics; methodology; neuropsychology; shelters; minorities; psychometric validity

\section{Introduction}

Homelessness is a major issue in many countries and large cities. According to the European Federation of National Organizations Working with the Homeless (FEANTSA), homelessness can be subdivided into being 'roofless' (i.e., without shelter of any kind) or 'houseless' (with only a temporary place to sleep, such as an institution or homeless shelter), while 'housing exclusion' describes those whose housing is insecure (e.g., with an ongoing threat of eviction or domestic violence) or inadequate (such as in unfit housing, or caravans on illegal campsites) [1]. In 2020, it was estimated that about 28,000 homeless and houseless people were living in Paris (France) [2]. This equates to over $1.3 \%$ of the total population of the city. Of these people, 3500 were roofless and up to 25,000 regularly stayed in shelters or hotel rooms [2].

Although the majority of people recorded as being homeless in Paris were not actually sleeping rough, the figures reveal the failings of the current service provision. Temporary 
accommodation is only supposed to provide an interim solution while people find more permanent lodgings. Instead, the reality is that such places are so saturated that people continue to be roofless or living in inappropriate structures because of a lack availability of suitable accommodation.

In addition, research has also shown that as the number of people in extremely precarious living situations rises, few are managing to break the cycle and move into regular housing [3]. This may be due to the multifactorial nature of homelessness [4-7] as well as a lack of political focus on addressing the causes of homelessness within national policies [8,9]. The phenomenon of living in temporary accommodation has been termed the "paradox of chronic emergency" [10]. Providing basic needs for this population, such as a roof or food, is essential but is also insufficient. Social rehabilitation can only be effective when the biopsychosocial causes of homelessness are also addressed [8,9,11].

Research into the psychological rehabilitation of homeless people is lacking in France. Social workers engaging with homeless people may also lack appropriate training to recognize the need for referral to a psychologist [12]. More data are essential, as well as more research into tackling homelessness from a psychological standpoint $[13,14]$. Such research into homelessness has, to date, only been carried out in a limited number of countries. In 2008, for example, a systematic review found 29 published studies of mental disorders in homeless people [15], of which the majority $(n=26)$ came from only four different countries, namely the USA, UK, Germany, and Australia. In 2019, another systematic review found 31 published studies but the majority $(n=22)$ again came from only four different countries, this time the USA, UK, Germany, and Canada [16]. Other authors have reported similar publication distributions for prevalence studies into cognitive disorders [17-21].

As a result of the considerable differences that exist between the countries' key services, particularly the accessibility of mental health care, availability of suitable housing stock, and rates of poverty, it is difficult to be sure that the results from such country-specific studies can be applied to the situation in France. Research into psychological aspects of French homelessness is needed. This is not only to ensure that existing, global data are relevant here but also to ensure that local research communities, service providers, and other key stakeholders are aware of the situation in their own backyard [13].

Despite possible limitations in the extrapolation of results, the methodologies and approaches used are invaluable: it has been reported that investigators avoid the inclusion of homeless populations within clinical trials due to practical concerns about conducting research with this population. Researchers such as Ojo-Fati et al. [22], for example, have published practical descriptions of their experiences, lessons learned, and challenges faced during the implementation of two large clinical trials into smoking cessation in homeless populations in the US.

Against this background, we wish to share our experiences from a psychology research project into homelessness. Our study context was different from the one described by Ojo-Fati and colleagues [22]: we were not describing clinical trials in the US, but rather an investigation into the cognitive profiles of people experiencing homelessness in France. Data collection from the preliminary phase of the work ended in December 2020 and results will be published in a future, dedicated paper. Here, we present our practical experience from the design and conduct of the pilot study: lessons learned, challenges encountered, and solutions implemented. We have also included a list of recommendations that we hope will be of use for the future, and much needed psychological studies on homelessness.

\section{Study Settings}

Study context. Studies have shown that homelessness is associated with several risk factors for developing cognitive disorders, such as traumatic brain injuries [4,23], neurodevelopmental issues [24,25], or alcohol consumption [26,27], and that the prevalence of cognitive disorders is higher in people experiencing homelessness [11,28]. Although 
research into mental disorders in homeless people has been carried out in France [29-31], there is a lack of data for cognitive disorders specifically from a French population.

The aim of our research was to describe the prevalence of cognitive disorders and risk factors for cognitive health problems among homeless people in Paris. In order to do this, our project was divided into two phases. The first phase, which is reported here, was a small-scale preliminary pilot study undertaken in Parisian shelters in November and December 2020 on a small sample of volunteers. The results from this pilot study allowed us to refine the method to be used in the larger-scale study.

Ethical considerations. Ethical approval was granted by the CPP Sud Ouest-OutreMer II for the pilot study.

Participants. To be eligible for inclusion in the preliminary study, participants had to be homeless according to French [32] and European [1] definitions. In practice, they had to be users of a shelter for homeless people. Participants had to be over 18 years of age, speak sufficient French to understand and to be easily understood, and able to consent to participation. People under guardianship or under the acute effect of psychoactive substances were ineligible for inclusion.

Recruitment. Recruitment was conducted between 1 November and 31 December 2020 at three centers for homeless people run by the same association. The first was an emergency shelter that offered medium-term accommodation to young women aged 18-25 years. The second was a medium-to-long-term stay shelter for homeless couples (corresponding, in French, to a "centre d'hébergement et de réinsertion sociale"). The third was a very short-term night shelter for men; it was only open overnight so that users had to leave in the morning and return in the evening.

The three centers were chosen because of their diverse user profiles (different ages, genders, and marital statuses) and institutional functioning. Each shelter represented different levels of housing stability. At each center, one member of staff acted as the point of contact; this staff contact introduced the researcher to service users and reminded participants about their meeting with the investigator $24 \mathrm{~h}$ before.

Recruitment was performed by first randomly selecting service users, and then determining if those chosen were eligible for inclusion. If they were, these individuals were approached by the investigator and the staff contact to introduce the study to them. If the individual agreed to participate, they were provided with an information letter and a meeting was planned for the following week to allow them time for reflection on the study and their participation.

During the two months, $n=39$ people were randomly selected. Of this initial population, $n=18$ were ineligible, mostly because of poor spoken French (discussed in further detail later in the paper). A further $n=7$ declined the invitation to participate. Therefore, $n=14$ individuals were recruited: $n=5$ from the women's shelter, $n=4$ from the couples' shelter, and $n=5$ from the men's shelter. Nine participants fully completed the multiple components of the pilot study, as discussed in greater detail later.

Data collection. The data collection meeting began with the investigator (a licensed psychologist) answering any questions from the participants before they signed the informed consent form.

Secondly, a semi-structured interview was used, with questions on the following three life domains: childhood, young adulthood, and professional life. Questions also covered medical history, including specific questions on neurological disorders and drug use, mental health, and the participant's psychological resources (such as resilience skills).

Each participant was then asked to complete the self-reported version of the Brain Injury Cognitive questionnaire or BICoQ, which contains 25 questions on potential symptoms related to brain injuries [33].

Finally, cognitive functions were assessed using several validated and standardized tests chosen for their psychometric properties and their ease of use. The functions assessed included executive functions, working and episodic memory, speech, praxia, visualconstructive functioning, and theory of mind. 
Follow-up session. For each participant, a follow-up session was organized one week after the first meeting. The aim of this second meeting was threefold.

(1) To determine if there were any negative effects of the first meeting since they had been interviewed about their personal lives and had undergone a relatively long cognitive assessment. If this was the case, the investigator was able to provide information and reassurance to reduce these effects.

(2) To collect the opinions of the participants regarding the questions, assessment, and tests used in the first meeting, and to find out if they had any recommendations that could be used for a future larger, phase 2 study.

(3) To provide the participants with feedback about their own cognitive profiles in order to suggest individualized interventions for them such as psychological interventions, or further neurological investigations.

\section{Obstacles and Solutions during the Study}

\subsection{Homeless Shelters Acting as a Host Venue for a Psychology Study}

Reluctance to participate by service care providers. The first obstacle we encountered to participant recruitment for our study was the reluctance of some associations, services, or staff members to introduce their service users to the research. Staff were concerned about the specific meaning and purpose of the study. They were unsure about how the results would be used and could be interpreted; there was anxiety that the research might result in a 'pathologization' of homelessness, which would only increase the stigmatization of this population. Care providers were also concerned that the tests used might have a negative impact on already-vulnerable people if participants 'failed' the cognitive tests or felt that they were being used as "guinea pigs".

We believe that some of these concerns were based on common misunderstandings that exist in France on the dichotomous roles of social and medical services in providing care for the homeless population $[12,34]$. Shelter staff members are not trained in psychology or psychiatry, and they do not have the time or resources to provide such care. Homeless institutions, for example, frequently complain that psychological and psychiatric services are not sufficiently present onsite $[12,13]$. As a result, social service and shelter staff do not have the opportunity to understand the role of a psychologist or psychiatrist because they are not present when the interventions occur. It is perhaps for this reason that psychiatric services frequently report receiving inappropriate referrals from homeless shelters for individuals who actually require social, rather than psychiatric, interventions [12]. Furthermore, psychologists and psychiatrists lack understanding of the particularities of the homeless population and do not always consider their needs sufficiently, for example when organizing discharge after a period of hospitalization [12].

The first homeless support association we contacted declined our request to participate in the project. After our initial disappointment we decided upon a different approach for the second association. We redoubled our efforts to provide sufficient information and reassurance to all staff whose assistance we would need. We organized meetings with the staff from each center to present the study to them, to describe its practical organization, and, crucially, to be available to answer their questions. This step was important; it created transparency regarding our motivations, it showed our commitment, and it personally introduced the team members to the staff. This time strengthened the partnership between the research team and the association, it reassured staff, and answered their concerns.

In our important pre-research discussions with the association, we dedicated significant time to explaining that our work aimed to decrease stigmatization rather than staff's feelings that it might increase it. We wanted to reveal 'invisible disabilities', to identify issues that could be addressed. We explained how we did not seek to pathologize homelessness as a single problem but instead wanted to clarify not only the individual circumstances that lead to homelessness but also people's resources and strengths which could also be used to help them break the cycle of homelessness by offering targeted support that played to these strengths. 
Additionally, we spent time speaking with staff about their concerns that the assessments to be undertaken by participants could have a negative impact. To reassure staff on this point, we described the experimental methods that we planned to use in detail. We emphasized the empowerment that this knowledge about themselves would give to participants.

Finally, we also found that we needed to highlight the direct benefits that would be brought to the association and/or the services as a result of the work. The staff accepted our offer to present our preliminary results to the teams at the end of the project, before the work was written up for publication. We promised to produce a detailed but accessible account of our findings in the format of an information booklet. This booklet would summarize the key findings that were relevant for the service in order to improve overall care provision.

We would strongly encourage all researchers to engage thoroughly with their chosen service provider. This time spent doing this is an investment in stronger relations, delivering benefits to all parties. Other authors have also noted that this kind of approach helps stakeholders to understand the role that research plays in the development of their practice. Gaetz noted that engaging with researchers should be seen as an opportunity for the service, rather than only as a constraint [35]. Brown also concluded that research should be neither isolated from clinical practice psychology nor from the third sector. By working hand-in-hand with service staff and commissioners, research can bring practical benefits to structures and, by extension, to homeless people themselves [36].

Practicalities. It is important to remember that it is the hosts who best know the situation and reality in the field. We quickly discovered that there were practical issues which needed to be dealt with in advance of the recruitment and interviews. It was invaluable to our work that our host organization were not passive partners in our research (i.e., that they simply agreed that we could interview their service users) but that they were genuine partners in the study and its outcomes. As genuine partners, this means that the host organization was involved in the practical organization of the study.

One important aspect of our research partnership was having a named member of the association's staff as a contact. This provided one person that the research team and staff of the host organization could liaise with to provide or receive up-to-date information. The staff contact also facilitated the organization of individual meetings with potentially eligible participants on a one-to-one basis. These individual meetings eliminated the need to organize a public meeting with all service users to try and recruit them. While this group approach would have been perhaps easier for the research team, it would have involved more preparation for the staff and would have required them all to be able to answer the service users' questions in detail once the researchers had left. Finally, the presence of the staff contact during the first meeting with users also fostered better relations and encouraged trust between researchers and service users.

A second important reason to involve the staff of the service in the project planning was due to the limits of the service premises themselves. It was essential to have a private space to conduct the meetings. Many shelters in Paris, however, are not located in dedicated, purpose-built clinical buildings with private consulting rooms. Instead, they often occupy repurposed venues, due to both their city-center location and because the cost of such buildings as venues for service delivery is comparatively lower than purpose-built spaces. Two of the three shelters in our study were previously hotels, and the third was formerly a cultural center. We therefore had to negotiate with staff for the use of an office at a mutually convenient time to ensure meetings for the study did not clash with other service needs.

Consideration of some of the practicalities of the design and delivery of our study led us to some clear observations. Foremost, service providers should not be seen simply as facilitating access to the population of interest but as equals who provide invaluable practical organization and insights into their service and their service users. Researchers must involve shelter staff as genuine partners in their research from the very start. All employees of the shelters, including care commissioners, social workers, and the volunteers 
on the ground can offer practical support as well as insight and suggestions to improve the research. Researchers must be open to sharing their expertise without prejudice towards staff who may not have received their own particular training since they will, once informed about the project, be able to tell them how the project could be implemented and who in their shelters can benefit from the research process and from its results.

\subsection{Administrative Organization}

Obtaining ethical approval. Following legislation changes in 2016, in France, certain psychology studies must be evaluated by an independent ethics committee called the CPP (Comité de Protection des Personnes) that oversees the protection of participants [37]. Our study fell into this category because it involved the use of clinical tests in a vulnerable population. We therefore applied for ethical approval to a CPP. Our first application was returned for further justification of the fact that (1) the population was not a patient population and (2) there was a risk of psychological decompensation associated with participation. We believe that these concerns arose from a lack of familiarity with the homeless as a research population. Once we clarified our aims and methodology the CPP approved our study. For information, here are the justifications presented in our second application in case they are of help to future researchers applying for ethical permission:

- Homeless people have unmet medical needs that need to be better described and documented through research [30], even if they do not correspond to a medically defined patient population. Homelessness is rarely a choice and relatively few individuals who are homeless choose to not use food banks, shelters, or other social and professional care services [2]. Although being homeless is not defined as a medical condition, the homeless population is known to have a higher risk of serious health issues. Homelessness is listed in the chapter "Factors influencing health status and contact with health services" of the International Classification of Diseases [38].

- Research into the risk factors and causes of homelessness supports practical work to help people to improve their situation. Homelessness is rarely explained by one simple cause, such as the loss of a job or the break-up of a single relationship. Research has shown that it is multifactorial and due to an accumulation of issues, such as socioeconomic disadvantage, low educational attainment, lack of a familial support network [39,40], mental health issues [40,41], and childhood adversity [25,42].

- Homeless research should not always focus on psychosis. Research has shown that only $13 \%$ of homeless people in Paris have been diagnosed with a psychotic pathology [29]. While this prevalence is high, so too are the figures in the same population for alcohol dependence (20\%) and mood disorders (22\%).

- Psychological interviews and cognitive tests are not generally considered to constitute a major risk for decompensation. Psychological researchers are trained to conduct interviews, and this includes techniques to reduce participant anxiety and avoid decompensation. Furthermore, the homeless people interviewed in their shelters are not isolated, they do have social resources, and thus we believe the study presents a low risk of decompensation.

Inclusion of people without identification documents. According to French law, participants in biomedical research must be registered in the social security system. Participants included as controls, rather than experimental subjects with a disease or disorder, must also be informed that their details will automatically be included in a national register of healthy volunteers.

We were concerned that this could lead to recruitment bias due to both the exclusion of potential subjects and a reluctance by others to participate. Data from 2012, for example, showed that $14 \%$ of the French homeless population and $88 \%$ of immigrants were not registered in the health care system [43]. We therefore applied to the CPP for an exemption to allow us to both include individuals not registered in the social security system and not obliged to register participants as healthy volunteers. We justified this by explaining that it would allow the research to access otherwise inaccessible eligible individuals who would 
be unable or unwilling to participate either for administrative reasons or for fear of being tracked by the State. This was accepted.

\subsection{Creating an Adapted Protocol for a Specific Population}

The aim of the preliminary study was to test the validity of our methodology to assess the prevalence of cognitive disorders and risk factors for cognitive health problems among homeless people in Paris. To our knowledge, this is the first such study in France and so without precedent, accordingly some protocol aspects required particular attention to ensure it was relevant for the homeless population we wished to study. This section outlines these key protocol aspects.

Language and cultural barriers. The most frequent reason for people being ineligible to participate was an insufficient level of spoken French. In 2012, it was found that $20 \%$ of homeless people did not speak French [44]. Given that migrants represented $56 \%$ of the adult homeless population in France [44], and that this proportion is likely to have grown since the European migrant crisis of 2015 [45], a considerable proportion of homeless people are unlikely to speak adequate French. It is important that when undertaking cognitive evaluation of the homeless, both language barriers and cultural sensitivities are considered since they are likely to represent significant portions of the population being studied. Language and cultural backgrounds influence neuropsychological assessments and play roles on different scales.

Language conditions the practical use of tests. Indeed, most tests require verbal communication, even when they evaluate visuospatial situations (because, at a minimum, instructions have to be read/understood). Thus, tests cannot be realized if the investigator and the participant do not have a sufficient grasp of the same language. For this reason, the ability to speak French was an inclusion criterion. However, for more inclusivity, we did not set not being able to read and/or write French as an exclusion criterion. This choice was also guided by the fact that most of the study did not require literacy (the whole interview, most cognitive tests, and the follow-up session). For some tests, a small amount of literacy was required (e.g., to read instructions) and the investigator helped the participants when needed. Only a small number of tests had to be abandoned because they required more advanced literacy skills that could not be compensated for by the investigator. Therefore, the preliminary phase helped to identify how much language (oral and written) was a barrier for the future main study. We were able to see which tests were not feasible in their standard conditions (without the help of the investigator, for example) and would need to be replaced by alternative tools.

Cultural background influences the relevance of using tests that have been validated for another cultural background than that of the participant. Indeed, it is known that early neurodevelopment differs between cultures [46], meaning that evaluating foreigners with tests developed for the French population may not be appropriate, because French standardization would not match with the cognitive functioning of the person. Some solutions are used in daily practice to address this limitation (e.g., adapting some items that are influenced by cultural background, or using non-verbal tests), but these solutions are unfortunately insufficient to guarantee a valid assessment, without any risk of false positive results. When possible, the use of tools which have been specifically developed for foreigners is a good option (for an example, see [47]) but such alternatives are rare. As Lecerf [46] suggested for the assessment of intellectual performance, cultural biases cannot be removed from evaluations. A preferred option is to evaluate how much culture influences the results of tests. As an example, Lecerf describes the C-LIM classification (Flanagan et al., cited by [46]), which classifies each subtest of intelligence assessments according to how much they are influenced by language and cultural background. Thus, it is possible to determine if a given participant fails more "cultural-dependent" tests than "cultural-independent tests". If this is the case, observed difficulties might be due to cultural background (and are then false positive results). However, if cultural-independent subtests are also failed, this is more likely to indicate true cognitive disorders. This type of 
classification could be useful, not only for psychometric tests of intelligence, but also for tests of cognitive function.

Meeting duration and frequency. It is very important that all study meetings are planned to suit the homeless population being studied. In particular, those who are being interviewed in short-term accommodation, such as overnight shelters, may already have limited time to eat and sleep once they arrive and before they have to return to the streets the following morning. It should be remembered that for the participant, granting the researcher long or multiple interviews may present a considerably greater personal inconvenience than that experienced by a researcher who has to return to the shelter several times or wait until late in the evening in order to collect their data.

In the present study, each first meeting lasted for about $3.5 \mathrm{~h}$. This was composed of a one-hour interview, two hours of cognitive assessments and two $15 \mathrm{~min}$ breaks. This was too long for some participants to remain focused. As a result, we adapted the schedule for 5 of the 14 participants and they completed the cognitive evaluation over two meetings. This meant that they actually participated in three meetings.

The reasons why participants failed to remain focused for the duration of the meeting was often due to fatigue. The night shelter, for example, did not open until 8pm and so meetings there in particular were in the evening or at night. In addition, the research was undertaken during November and December, when the average temperature in Paris was between $7{ }^{\circ} \mathrm{C}$ and $12{ }^{\circ} \mathrm{C}$ during the day. Participants usually spent the day outside and were, understandably, easily tired. Since they had to leave the shelter in the morning they needed to sleep. Other participants were unable to complete one long meeting either because they arrived late, or because they had personal engagements afterwards. In these circumstances, two meetings were found to be a good solution. Thanks to the flexibility and accommodation of the service staff and facilities, the researchers were able to organize two meetings when necessary and so the majority of recruited participants did complete the whole evaluation.

Of the $n=14$ participants, $n=11$ completed the whole cognitive evaluation component of the study. The other $n=3$ failed to attend the meeting and did not respond to our proposals to reschedule.

Reflecting on our learnings about participation in a study of this nature, we feel that where multiple meetings are necessary to collect data, follow-up sessions should be held as close as possible to the first meeting to maintain the participants' availability. In this study, even though the second meeting was short (less than an hour), it was planned for at least a week after the first meeting, and this seemed to increase the risk of attrition. In the present study, of the $n=11$ who completed the whole assessment, $n=2$ were lost to follow-up. As a result, from an original study population of $n=14$, we only had complete datasets from $n=9$ participants.

Proxy checking for self-reported data. The present study did not involve access to participants' general histories or other medical evaluation that could be compared with our neuropsychological evaluation data. Instead, it relied on self-reporting to determine risk factors for cognitive disorders. Although the interviews did not provide quantitative data, they did provide information that may be overlooked by medical assessments, such as cognitive disorder risk factors related to issues from participants' childhoods including trauma or learning disabilities. Interviews also provided a more flexible and targetable approach than questionnaires for gaining such information in a heterogenous population.

Use of self-reported tools and interviews, however, presents some disadvantages. Presence of cognitive disorders, for example, can affect the accuracy of self-report, thus responses must be cautiously interpreted [21]. A solution to these challenges is to seek a proxy opinion from the staff contact at the shelter. This is yet another good reason to integrate shelter staff into the research project since they usually have good knowledge of the backgrounds and individual circumstances of service users and are able to act as reliable informants or obtain a second opinion on the participant's self-report from a colleague. 
Unintended consequences for the participants. There were no data available to indicate whether or not participants would be at increased risk of negative feelings (such as anxiety or a loss of self-confidence) following the interviews. Although our professional opinion was that they would not, the absence of in-house doctors or psychologists at the shelters meant that follow-up would be difficult. Therefore, to verify that unintended consequences from participation in the protocol did not happen, and to provide support to participants if necessary, we purposefully designed the study with a second, follow-up meeting. This meeting was presented as a chance for participants to provide feedback on our method, but it also conveniently served as an opportunity for the researchers to assure themselves that subjects had not be adversely affected by their participation.

Feedback sought and received on the adaptation of our research protocol to meet the specific needs of the study population (allowing for multiple meetings with participants to reduce the impact of fatigue and burdens placed on participants because of the length of assessments) yielded positive commentary. Participants noted that they enjoyed both completing the cognitive exercises and learning about themselves. Others described how they appreciated feeling useful by taking part in research that had a clinical application. Some participants also said that they benefited from meeting with a psychologist; even the single participant who reported anxiety related to cognitive test results indicated that the follow-up session was reassuring and had made her feel better.

\section{Discussion}

In this article, we have described our experience of implementing a psychological study in three different homeless shelters in France. We have listed the main difficulties that we encountered in the project and also described their context and the solutions we found to overcome obstacles.

The work has clearly demonstrated that a homeless population presents particular, although by no means insurmountable, challenges for psychology researchers. One of the most important points in overcoming any challenges in conducting this research is the necessity for the researchers to take the time and make the effort to foster a strong relationship with the services from which participants are to be recruited. Service staff and care providers are vital partners in the research and should not be considered simply as study hosts. Their experience with their clients and homelessness in their local area is invaluable: it helps to focus the study on the right issues, build trust with service users, and allow direct application of the acquired knowledge to the care of the target population. It may be that services are unfamiliar with psychologists and psychology research. It is, therefore, incumbent on the researchers to explain and adapt themselves to services and to the field.

More research is needed on homelessness in France. Not enough is known about the needs and specificities of homeless people generally, and especially in areas related to psychology. Services supporting homeless people can benefit from the results of research studies such as this pilot project, ultimately informing evidence-based practice. Although research on mental health, cognition, or psychological interventions for homeless people is conducted in a few countries, it remains largely under-represented worldwide, and the presence of psychologists in homeless shelters needs to be encouraged in countries, including France. Access to mental health care should be also facilitated for those experiencing rooflessness (i.e., living in the street) because their needs may be even greater. In 2010, the American Psychological Association published a report on the role of psychologists in helping people without homes [48]. The report recommended integrating a theoretical and practical training module on homelessness for all psychology students. We support this recommendation; it supports our belief that increased research is needed to address the lack of psychologists in the field. As long as psychology studies of homelessness remains a marginal subject in some countries then it will continue to make research there more difficult. It will make it harder for projects to obtain funding or ethical approval since the 
cost-benefit balance or feasibility seems less obvious for homelessness than for other, better known, subjects.

A further important observation from our work concerns the structure and processes of research with homeless people, especially research protocols. Protocols for psychological studies on homelessness require scrupulous attention to detail because homelessness is a very difficult situation to assess: its heterogeneity makes it impossible to apply a 'one size fits all' method. Instead, researchers have to redouble their creativity to elaborate protocols covering each individual situation and with the most relevant measurements among the wide range of possible variables. These methodological choices are also conditioned by the difficulty in undertaking studies over a prolonged period and the fact that tumultuous circumstances of homelessness present some challenges for the conduct of research. However, these factors can also be drivers for methodological and practice-based innovation.

This design and methodological reflection on our pilot study show one example of where innovation is clearly needed: developing adaptable and appropriate tests and measurements (such as neuropsychological assessments) for homeless people. At the moment, existing tools can be difficult to apply and interpret because their context of validation does not match with the conditions of use in a homeless population. These obstacles should not discourage future studies. Researchers should instead find new ways to observe phenomena, including those which are more challenging to capture. Alternatives to the standard measurements exist and could be introduced and used to make the psychometric assessments more accurate and more adapted to the specificities of homelessness. Rigorous literature reviews on such methodological questions and the development of dedicated psychometric tools may ease the challenges of future research in the field. Before further and larger studies are rolled out, exploratory phases should be undertaken, evaluated, and reflected upon to identify design, methodological, and delivery levers to adapt.

For all the reasons identified, more methodological papers such as Ojo-Fati's [22] are needed. Reports of local experiments and research experience in the field raise the profile of a marginalized population and important areas of research to build better understanding and responses. Future research should build on such studies to further refine methodological choices to avoid what has been reported as unfruitful. Moreover, as noted earlier, geographical discrepancies related to institutional offerings and to factors contributing to homelessness pathways for individuals may limit the generalizability of data but this can only be known for sure if there are a wide variety of local data published which will allow identification and understanding of both common traits and regional, national, or international differences.

As a conclusion, we propose the following recommendations for the initiation of psychological studies with homeless people:

1. Empowering participants. People in shelters often do not feel in control of their life. Asking participants for their own recommendations to improve the design and delivery of research, asking for their feedback about protocols, and offering a followup session to discuss the results of their evaluation are solutions that can support positive participant experiences.

2. Empowering shelter staff. Associations should be considered as partners in our research. They help in reinforcing participants' trust towards the researchers and their knowledge of service users is useful for the practicalities of research and for understanding the backgrounds and circumstances of service users, including clinical needs. Staff are also able to both use and apply the results of research, and they must become confident and familiar with psychological studies in order to do this.

3. Flexibility in the implementation of research. Despite the fact this sounds true for all research and/or all psychological interventions, this is particularly important for the work with homelessness. Services often have limited resources and are not used to hosting scientific studies. Furthermore, study participants are first of all service users and unlike patients in hospitals, they usually stay where they will be approached for 
their research participation. As external researchers, we must respect their routine and their privacy, and we should adapt our data collection approaches as much as possible when it does not fit the constraints presented by the realities in the field.

4. Sharing professional experience and methods. While there are some psychological interventions available to homeless services, these clinical services often lack visibility, not only in the wider research field but also in services themselves. Sharing results with the scientific community from all stages of a research project, including the exploratory phase and both personal and professional experience, helps guide future methodological choices. It also allows other researchers to identify specificities that only apply to local situations. Providing the results of studies back to participants and stakeholders is a critical part of the research process that must not be overlooked. This step can be important in evolving evidence-based practice within services.

5. Innovation in the use of measurement tools. Most psychometric tools are not validated for use with homeless populations and this means that they cannot be compared with other data. Where possible, data collected from general population tools should be interpreted in new ways, such as through intragroup comparison or longitudinal descriptions. Even if they remain comparatively rare, alternative tools more adapted to the homeless people do exist (e.g., dedicated tests for allophone subjects) and the more they are used, the greater their inter-study reliability will become.

Author Contributions: Conceptualization, F.B., M.-C.C., G.C. and C.V.-A.; project administration, F.B.; investigation, G.C.; resources, M.C.; supervision, M.-C.C. and C.V.-A.; writing-original draft preparation, G.C.; writing-review and editing, F.B., M.-C.C., M.C. and C.V.-A. All authors have read and agreed to the published version of the manuscript.

Funding: This research received no external funding.

Institutional Review Board Statement: The study was conducted according to the guidelines of the Declaration of Helsinki, and approved by the Institutional Review Board of Sud-Ouest et Outre-Mer II (ID-RCB number: 2019-A02600-57; date of approval: 5 October 2020).

Informed Consent Statement: Informed consent was obtained from all subjects involved in the study. Data Availability Statement: Not applicable.

Acknowledgments: We would like to thank the Groupe SOS Solidarite and its participating homeless services who hosted and assisted the study. We also thank services users who gracefully accepted to take part in the study. We wish to thank Jennifer Dandrea Palethorpe and Johanna Robertson for language assistance.

Conflicts of Interest: The authors declare no conflict of interest.

\section{References}

1. FEANTSA ETHOS-European Typology on Homelessness and Housing Exclusion. Available online: https://www.feantsa.org/ en/toolkit/2005/04/01/ethos-typology-on-homelessness-and-housing-exclusion (accessed on 16 March 2021).

2. Atelier Parisien d’Urbanisme. Les Personnes en Situation de rue à Paris la Nuit du 30-31 Janvier 2020-Analyse des Données Issues du Décompte de la 3e Edition de la Nuit de la Solidarité; APUR: Paris, France, 2020; p. 90.

3. Yaouancq, F.; Lebrère, A.; Marpsat, M.; Régnier, V.; Legleye, S.; Quaglia, M. L’hébergement des sans-domicile en 2012. Insee Prem. 2013, 1455, 4.

4. Anderson, I.; Christian, J. Causes of Homelessness in the UK: A Dynamic Analysis. J. Community Appl. Soc. Psychol. 2003, 13, 105-118. [CrossRef]

5. Mabhala, M.A.; Yohannes, A.; Griffith, M. Social Conditions of Becoming Homelessness: Qualitative Analysis of Life Stories of Homeless Peoples. Int. J. Equity Health 2017, 16, 150. [CrossRef]

6. Susser, E.; Moore, R.; Link, B. Risk Factors for Homelessness. Epidemiol. Rev. 1993, 15, 546-556. [CrossRef] [PubMed]

7. Brown, R.T.; Goodman, L.; Guzman, D.; Tieu, L.; Ponath, C.; Kushel, M.B. Pathways to Homelessness among Older Homeless Adults: Results from the HOPE HOME Study. PLoS ONE 2016, 11, e0155065. [CrossRef] [PubMed]

8. Kourachanis, N. Southern European Welfare States and Homelessness: Portugal and Greece. Hous. Care Support 2019, 22, 114-128. [CrossRef]

9. Bruneteaux, P. L'hébergement d'urgence à Paris ou l'accueil en souffrance. Soc. Contemp. 2006, 63, 105. [CrossRef]

10. Rullac, S. L' urgence de la Misère SDF et SAMU Social; Ed. des Quatre chemins: Paris, France, 2004; ISBN 978-2-84784-113-8. 
11. Solliday-McRoy, C.; Campbell, T.C.; Melchert, T.P.; Young, T.J.; Cisler, R.A. Neuropsychological Functioning of Homeless Men. J. Nerv. Ment. Dis. 2004, 192, 471-478. [CrossRef]

12. Baronnet, J.; Alberghini, A. Aux portes de la rue ou quand les institutions produisent l'exclusion: Les sortants d'hôpitaux psychiatriques. Rech. Soc. 2018, 4, 6-91. [CrossRef]

13. Maguire, N.; Ritchie, C. Clinical Psychology: A Rare and Essential Resource in Commissioning Quality Services for Homeless People. Clin. Psychol. Forum 2015, 265, 23-27.

14. Backer, T.E.; Howard, E.A. Cognitive Impairments and the Prevention of Homelessness: Research and Practice Review. J. Prim. Prev. 2007, 28, 375-388. [CrossRef] [PubMed]

15. Fazel, S.; Khosla, V.; Doll, H.; Geddes, J. The Prevalence of Mental Disorders among the Homeless in Western Countries: Systematic Review and Meta-Regression Analysis. PLoS Med. 2008, 5, e225. [CrossRef] [PubMed]

16. Ayano, G.; Tesfaw, G.; Shumet, S. The Prevalence of Schizophrenia and Other Psychotic Disorders among Homeless People: A Systematic Review and Meta-Analysis. BMC Psychiatry 2019, 19, 370. [CrossRef] [PubMed]

17. Burra, T.A.; Stergiopoulos, V.; Rourke, S.B. A Systematic Review of Cognitive Deficits in Homeless Adults: Implications for Service Delivery. Can. J. Psychiatry 2009, 54, 123-133. [CrossRef] [PubMed]

18. Depp, C.A.; Vella, L.; Orff, H.J.; Twamley, E.W. A Quantitative Review of Cognitive Functioning in Homeless Adults. J. Nerv. Ment. Dis. 2015, 203, 126-131. [CrossRef] [PubMed]

19. Chevreau, G.; Castillo, M.-C.; Vallat-Azouvi, C. Une personne SDF sur 10 souffre de troubles cognitifs: Que sait-on de ces troubles? Une revue de littérature sur la cognition des personnes SDF. L'Encéphale 2019, 45, 424-432. [CrossRef] [PubMed]

20. Fry, C.E.; Langley, K.; Shelton, K.H. A Systematic Review of Cognitive Functioning among Young People Who Have Experienced Homelessness, Foster Care, or Poverty. Child Neuropsychol. 2017, 23, 907-934. [CrossRef] [PubMed]

21. Stone, B.; Dowling, S.; Cameron, A. Cognitive Impairment and Homelessness: A Scoping Review. Health Soc. Care Commun. 2018. [CrossRef]

22. Ojo-Fati, O.; Joseph, A.M.; Ig-Izevbekhai, J.; Thomas, J.L.; Everson-Rose, S.A.; Pratt, R.; Raymond, N.; Cooney, N.L.; Luo, X.; Okuyemi, K.S. Practical Issues Regarding Implementing a Randomized Clinical Trial in a Homeless Population: Strategies and Lessons Learned. Trials 2017, 18. [CrossRef]

23. Stubbs, J.L.; Thornton, A.E.; Silverberg, N.D.; Sevick, J.M.; Barr, A.M.; Honer, W.G.; Panenka, W.J. Traumatic Brain Injury: An Underappreciated Determinant of Health in Individuals Who Are Homeless or Precariously Housed. World Neurosurg. 2020, 137, 474-475. [CrossRef] [PubMed]

24. Oakes, P.M.; Davies, R.C. Intellectual Disability in Homeless Adults: A Prevalence Study. J. Intellect. Disabil. 2008, 12, 325-334. [CrossRef]

25. Pluck, G.; Lee, K.-H.; David, R.; Macleod, D.C.; Spence, S.A.; Parks, R.W. Neurobehavioural and Cognitive Function Is Linked to Childhood Trauma in Homeless Adults: Childhood Trauma and Homelessness. Br. J. Clin. Psychol. 2011, 50, 33-45. [CrossRef]

26. Hurstak, E.; Johnson, J.K.; Tieu, L.; Guzman, D.; Ponath, C.; Lee, C.T.; Jamora, C.W.; Kushel, M. Factors Associated with Cognitive Impairment in a Cohort of Older Homeless Adults: Results from the HOPE HOME Study. Drug Alcohol. Depend. 2017, 178, 562-570. [CrossRef]

27. Fichter, M.M.; Quadflieg, N. Course of Alcoholism in Homeless Men in Munich, Germany: Results from a Prospective Longitudinal Study Based on a Representative Sample. Subst. Use Misuse 2003, 38, 395-427. [CrossRef] [PubMed]

28. Waclawik, K.; Jones, A.A.; Barbic, S.P.; Gicas, K.M.; O'Connor, T.A.; Smith, G.N.; Leonova, O.; Mathias, S.; Barr, A.M.; Procyshyn, R.M.; et al. Cognitive Impairment in Marginally Housed Youth: Prevalence and Risk Factors. Front. Public Health 2019, 7, 270. [CrossRef]

29. Laporte, A.; Vandentorren, S.; Détrez, M.-A.; Douay, C.; Le Strat, Y.; Le Méner, E.; Chauvin, P.; The Samenta Research Group. Prevalence of Mental Disorders and Addictions among Homeless People in the Greater Paris Area, France. Int. J. Environ. Res. Public Health 2018, 15, 241. [CrossRef]

30. Kovess, V.; Mangin Lazarus, C. The Prevalence of Psychiatric Disorders and Use of Care by Homeless People in Paris. Soc. Psychiatry Psychiatr. Epidemiol. 1999, 34, 580-587. [CrossRef] [PubMed]

31. Cougnard, A.; Grolleau, S.; Lamarque, F.; Beitz, C.; Brugère, S.; Verdoux, H. Psychotic Disorders among Homeless Subjects Attending a Psychiatric Emergency Service. Soc. Psychiatry Psychiatr. Epidemiol. 2006, 41, 904-910. [CrossRef] [PubMed]

32. Insee Définitions, Méthodes et Qualité. Available online: https://www.insee.fr/fr/information/2016815 (accessed on 14 October 2018).

33. Vallat-Azouvi, C.; Paillat, C.; Bercovici, S.; Morin, B.; Paquereau, J.; Charanton, J.; Ghout, I.; Azouvi, P. Subjective Complaints after Acquired Brain Injury: Presentation of the Brain Injury Complaint Questionnaire (BICoQ). J. Neurosci. Res. 2017, 96, 601-611. [CrossRef] [PubMed]

34. Laforcade, M. Rapport Relatifà la Santé Mentale; Agence Régionale de Santé-Nouvelle-Aquitaine: Bordeaux, France, 2016; p. 190.

35. Gaetz, S. Knowledge Mobilization as Design: The Case of the Canadian Homelessness Research Network. Sch. Res. Commun. 2014, 5. [CrossRef]

36. Brown, R. When It Comes to Working with People without Homes, Where Is Clinical Psychology? Clin. Psychol. Forum 2015, 265, 42-45.

37. Dupont, M.; Lebrun, P.-B. La recherche impliquant la personne humaine et les comités de protection des personnes. In Droit à L'usage des Psychologues; Les Ateliers du praticien; Dunod: Paris, France, 2019; pp. 265-269, ISBN 978-2-10-077621-4. 
38. World Health Organization QD71.0 Homelessness. Available online: https:/ icd.who.int/browse11/1-m/en\#/http\%3A\%2F\% 2Fid.who.int\%2Ficd\%2Fentity\%2F1325772378 (accessed on 16 February 2021).

39. Shelton, K.H.; Taylor, P.J.; Bonner, A. Risk Factors for Homelessness: Evidence From a Population-Based Study. Psychiatr. Serv. 2009, 60, 8. [CrossRef]

40. Sullivan, G.; Burnam, A.; Koegel, P. Pathways to Homelessness among the Mentally Ill. Soc. Psychiatry Psychiatr. Epidemiol. 2000, 35, 444-450. [CrossRef] [PubMed]

41. Muñoz, M.; Vázquez, C.; Koegel, P.; Sanz, J.; Burnam, M.A. Differential Patterns of Mental Disorders among the Homeless in Madrid (Spain) and Los Angeles (USA). Soc. Psychiatry Psychiatr. Epidemiol. 1998, 33, 514-520. [CrossRef]

42. Herman, D.B.; Susser, E.S.; Struening, E.L.; Link, B.L. Adverse Childhood Experiences: Are They Risk Factors for Adult Homelessness? Am. J. Public Health 1997, 87, 249-255. [CrossRef]

43. Dietrich-Ragon, P. Aux portes de la société française. Les personnes privées de logement issues de l'immigration. Population 2017, 72, 7-38. [CrossRef]

44. Mordier, B. Introduction de cadrage. Les sans-domicile en France: Caractéristiques et principales évolutions entre 2001 et 2012. Econ. Stat. 2016, 488, 25-35. [CrossRef]

45. Besozzi, T. Il y a de plus en plus de SDF. In Idées Reçues sur les SDF; Idées reçues; Le Cavalier Bleu: Paris, France, 2020; pp. 15-23, ISBN 979-10-318-0389-0.

46. Lecerf, T. Influence de la culture et du milieu socioéconomique sur les performances cognitives. Rev. Neuropsychol. 2014, 6, 191. [CrossRef]

47. Vanderaspoilden, V.; Fery, P. Validation et normalisation du test Brumory, une nouvelle tâche de mémoire épisodique picturale destinée à des populations non francophones ou peu scolarisées. Rev. Neuropsychol. 2018, 10, 159. [CrossRef]

48. American Psychological Association; Presidential Task Force on Psychology's Contribution to End Homelessness. Helping People without Homes: The Role of Psychologists and Recommendations to Advance Research, Training, Practice, and Policy; American Psychological Association: Washington, DC, USA, 2010; p. 53. 